\title{
GLICOPROTEÍNA-P, RESISTÊNCIA A MÚLTIPLAS DROGAS (MDR) E RELAÇÃO ESTRUTURA-ATIVIDADE DE MODULADORES ${ }^{\sharp}$
}

\author{
Paula C. Huber, Cintia H. Maruiama e Wanda P. Almeida* \\ Departamento de Química Orgânica, Instituto de Química, Universidade Estadual de Campinas, CP 6154, 13084-971 Campinas - SP, Brasil
}

Recebido em 6/1/10; aceito em 7/5/10; publicado na web em 24/8/10

\begin{abstract}
P-GLYCOPROTEIN AND MULTIDRUG RESISTANCE: STRUCTURE-ACTIVITY RELATIONSHIPS OF MODULATORS. Multidrug resistance, MDR is a major obstacle for cancer chemotherapy. MDR can be reversed by drugs that vary in their chemical structure and main biological activity. Many efforts have been done to overcome MDR based on studies of structure-activity relationships and in this review we summarize some aspects of MDR mediated by P-glycoprotein (P-gp), as the most experimentally and clinically tested form of drug resistance. The most significant MDR mechanisms revealed until now are shortly discussed. Physicochemical and structural properties of MDR modulators, measures of the MDR reversal, and QSAR studies are included.
\end{abstract}

Keywords: P-glycoprotein; multidrug resistance; modulators.

\section{INTRODUÇÃO}

Um dos maiores obstáculos para o sucesso da quimioterapia no tratamento de alguns tipos de neoplasias é o desenvolvimento, por parte das células tumorais, do fenômeno de resistência aos fármacos utilizados, não havendo resposta ao tratamento ${ }^{1}$ em cerca de $90 \%$ dos casos de câncer metastático. A resistência à quimioterapia é responsável pelo insucesso no tratamento. São reconhecidos dois tipos de resistência: a intrínseca, quando não há resposta mesmo no primeiro ciclo do tratamento, e a adquirida, quando surge no segundo ciclo (pós-terapia). ${ }^{2,3}$

Dada a importância do fenômeno de resistência procuramos nesta revisão reunir os resultados de vários estudos centrados na compreensão do fenômeno, no estabelecimento das relações estrutura-atividade de agentes moduladores da resistência e na evolução da geração destes agentes. As medidas de atividade moduladora e ensaios biológicos relacionados à investigação de novos moduladores também estão citados nesta revisão.

A resistência a múltiplas drogas (MDR) é um caso de resistência adquirida, observada em células tumorais, in vivo e in vitro, que consiste na ausência de resposta aos quimioterápicos, tanto àquele que foi utilizado inicialmente, quanto a outros empregados como alternativa. Uma das características mais intrigantes da MDR é que os quimioterápicos envolvidos não têm analogia estrutural nem farmacológica como, por exemplo, a vimblastina, que é um alcaloide bisindólico, e a doxorrubicina, que é uma antraciclina. ${ }^{4}$ A Figura 1 apresenta alguns quimioterápicos frequentemente envolvidos no fenômero de resistência a múltiplas drogas.

Este fenômeno de resistência a múltiplas drogas pode estar relacionado a uma série de fatores que não são plenamente compreendidos. Os principais fatores envolvidos são o transporte da droga através da membrana plasmática, ${ }^{5}$ alteração nas enzimas-alvo, ${ }^{6}$ alteração no metabolismo da droga, ${ }^{7}$ aumento na reparação do DNA ${ }^{8}$ e incapacidade para sofrer apoptose. ${ }^{9}$ Dentre estes, o primeiro é a principal forma de resistência e está relacionado com a presença de, no mínimo, três bombas que transportam ativamente o quimioterápico para fora da célula (bombas de efluxo). ${ }^{10}$

Linhagens celulares que apresentam fenótipo de resistência a

*e-mail: wanda@iqm.unicamp.br

* Artigo em homenagem ao Prof. Hans Viertler

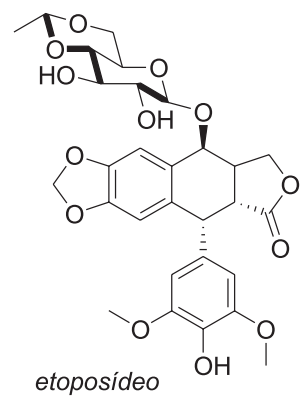

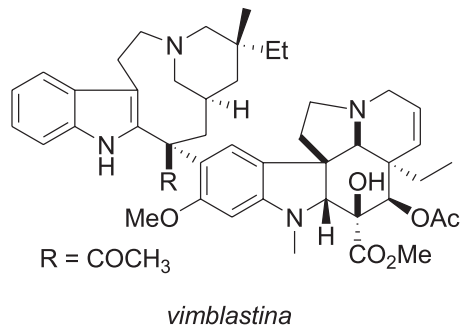

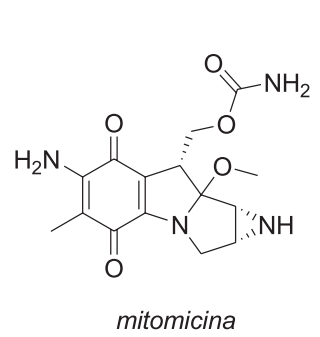<smiles>COc1cccc2c1C(=O)c1c(O)c3c(c(O)c1C2=O)C[C@@](O)(C(=O)CO)C[C@H]3O[C@@H]1OC[C@@H](N)[C@H](OC(N)=O)[C@H]1C(=O)O</smiles>

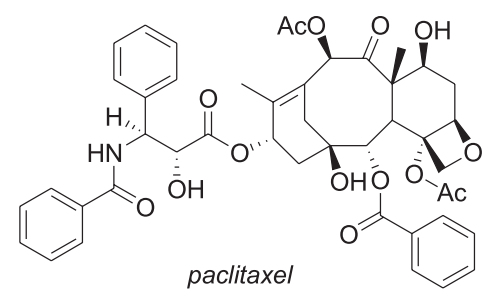

Figura 1. Quimioterápicos envolvidos no fenômeno $M D R$

múltiplas drogas apresentam baixa concentração intracelular do fármaco. ${ }^{11}$ Este fato está relacionado à amplificação gênica e/ou ao aumento da expressão de algumas proteínas pertencentes à superfamília ABC (ATP-binding cassette), cujo mecanismo de transporte é ATP-dependente. Em células humanas, os genes denominados mdr1 e mdr2 têm sido caracterizados, ${ }^{12}$ mas apenas o mdr1 está envolvido com o fenômeno de resistência múltipla a drogas. ${ }^{13} \mathrm{O}$ resultado da expressão do gene mdr1 em mamíferos é um tipo de glicoproteína, a glicoproteína-P (P-gp), que utilizando a energia proveniente da 
hidrólise do ATP, promove o efluxo do quimioterápico. ${ }^{14} \mathrm{O}$ mdr2 está envolvido no transporte de fosfatidilcolina. ${ }^{15}$

Outra proteína transportadora associada ao fenômeno de resistência múltipla a drogas é a MRP1. O genoma humano codifica somente duas P-gps, mas contém muitos genes relacionados à MRP1 $1{ }^{16}$ Esta proteína está localizada predominantemente na membrana plasmática de células, exibindo o fenótipo de resistência a múltiplas drogas. Atualmente, várias outras proteínas relacionadas à MRP1, incluindo nove humanas, foram descobertas em eucariotas. ${ }^{17,18}$

Apesar dos estudos apontarem várias proteínas relacionadas ao fenômeno de MDR, a MRP1, ou simplesmente MRP, e a glicoproteína-P são as mais importantes e conhecidas como promotoras do efluxo de quimioterápicos. ${ }^{19}$

Nos últimos anos, grandes esforços foram dispensados para se descobrir fármacos efetivos e atóxicos capazes de modular a resistência. Estes são chamados de reversores, moduladores da MDR ou, ainda, quimiossensibilizadores. As principais pesquisas estão concentradas na exploração direta do segundo uso de fármacos, ou seja, ativos farmacêuticos que são utilizados em função de uma atividade farmacológica principal, mas que têm como propriedade secundária a capacidade de reverter o fenômeno MDR. Quando estes fármacos não são empregados diretamente, eles ainda desempenham um papel extremamente importante como protótipos para o desenvolvimento de outros moduladores. Para exemplificar, pode-se citar o verapamil, nimedipina e amiodarona, utilizados como antiarrítmicos; trifluoperazina, como neuroléptico; quinina como antimalárico e a ciclosporina A, um imunossupressor (Figura 2), todos classificados como moduladores de primeira geração. No caso do verapamil, a estratégia para transformar o efeito secundário (MDR) no mais importante é o emprego do enantiômero $R$, como modulador da resistência. O eutômero para a atividade bloqueadora dos canais de cálcio, e por isso indicado como antiarrítmico, é o enantiômero $\mathrm{S}$, que é equivalente ao $R$ em atividade reversora da MDR. Entretanto, a potência deste último como bloqueador de canais de cálcio é muito inferior ao primeiro. $\mathrm{O}(R)$-Verapamil (dexverapamil) é um modulador de segunda geração. Nos demais exemplos, o segundo efeito (modulador da MDR) torna-se significativo pelo aumento da dose, o que leva também à exacerbação do efeito principal, tornando-os tóxicos para o paciente.

Esta amostragem, por si só, já indica a variedade estrutural dos moduladores, assim como os substratos da P-gp (Figura 1), o que torna um desafio o estabelecimento das relações entre a estrutura química e a atividade moduladora. O conhecimento destas REA (relações estrutura-atividade) é de grande importância no planejamento de novos moduladores. ${ }^{20}$ Para o sucesso deste planejamento, várias áreas de estudo precisam ser exploradas, destacando-se o conhecimento do alvo, no caso a bomba de efluxo, seu(s) sítio(s) ligante(s); o mecanismo de efluxo e, também, as propriedades físico-químicas e requisitos estruturais importantes para a ação moduladora.

\section{RESISTÊNCIA MEDIADA PELA GLICOPROTEÍNA-P}

A resistência a quimioterápicos promovida pela glicoproteína-P é a forma de resistência mais estudada in vitro, in vivo e na clínica e, consequentemente, um grande número de fármacos e compostos recentemente sintetizados são investigados pelas suas habilidades em afetar o fenômeno. A resistência está associada com uma expressão aumentada de uma proteína transmembrana chamada glicoproteína P (P-gp). A P-gp tem um peso molecular de aproximadamente 170 $\mathrm{kDa}$, é constituída por 1280 aminoácidos e se apresenta com duas sub-unidades, cada uma com 12 segmentos transmembrana (TM). Ela foi descoberta primeiramente em células tumorais ovarianas resistentes em hamsters chineses ${ }^{14}$ e detectada posteriormente em várias outras células tumorais resistentes em animais e humanos. ${ }^{21}$ A proteína também está presente na membrana celular de alguns te-<smiles>C=CC1CC2CCC1CC2C(O)c1ccnc2ccc(OC)cc12</smiles><smiles>COCCOC(=O)C1=C(C)NC(C)=C(C(=O)OC(C)C)C1c1cccc([N+](=O)[O-])c1</smiles><smiles>CCCCc1oc2ccccc2c1C(=O)c1cc(I)c(OCCN(CC)CC)c(I)c1</smiles><smiles>CN1CCN(CCCN2c3cc(C(F)(F)F)ccc3SC3C=CC=CC32)CC1</smiles>

Amiodarona<smiles>[R]c1nc(N2CCCCC2)c2nc([R])nc(N3CCCCC3)c2n1</smiles>
Dipiridamol: $\mathrm{R}=\mathrm{N}\left(\mathrm{CH}_{2} \mathrm{CH}_{2} \mathrm{OH}\right)_{2}$

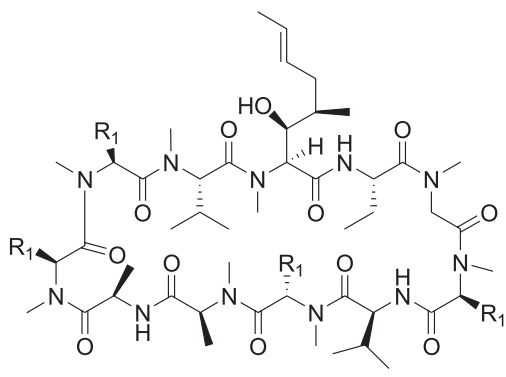

Ciclosporina A: $\mathrm{R}_{1}=\mathrm{CH}_{2} \mathrm{CH}\left(\mathrm{CH}_{3}\right)_{2}$

Figura 2. Moduladores da MDR de $1^{a}$ geração

cidos normais, por exemplo, rins, fígado, cólon, endométrio de útero e células endoteliais da barreira hemato-encefálica. A habilidade da P-gp em bombear compostos funcionalmente ativos do cérebro para a corrente sanguínea representa um mecanismo importante pelo qual o sistema nervoso central controla o periférico..$^{22,23}$

As variedades estrutural e funcional dos substratos e moduladores da P-gp levantam a questão de como funciona esta proteína e como pode ser explicada a ampla especificidade dos substratos/ moduladores. As perguntas tornam-se ainda mais complicadas quando é considerado o fato de que alguns moduladores (verapamil, ciclosporina A, diltiazem, etc.) se ligam a esta proteína e por ela são transportados, enquanto que outros moduladores (por exemplo, progesterona) que, mesmo se ligando a esta proteína e bloqueando o transporte dos agentes citotóxicos para fora, não conseguem ser transportados por ela.

Vários modelos já foram propostos para explicar o mecanismo de efluxo promovido pela $\mathrm{P}$-gp. Um dos modelos mais populares é o denominado "aspirador hidrofóbico", ${ }^{18,24}$ no qual as duas sub-unidades da P-gp formam um único canal de transporte, pelo qual o fármaco é expelido, quer seja na sua forma neutra, quer seja na forma carregada. Uma adaptação deste modelo foi proposta por Stein, ${ }^{25}$ que considera a ocorrência de mudanças conformacionais na proteína através da hidrólise de ATP. Estas mudanças conformacionais levariam à abertura de um "canal" pelo qual o quimioterápico seria expelido.

Higgins ${ }^{26}$ propôs, em 1994, que a ação da P-gp é semelhante ao de uma flipase. Atualmente, o modelo mais aceito é o que mescla as propostas de Higgins e Stein (Figura 3): o substrato é particionado da 
bicamada lipídica para uma parte mais interna da proteína, chamada de "folheto interno", entrando em um bolsão que constitui o sítio ligante. O ATP liga-se ao NBD (sítio de ligação de nucleotídeo), causando uma grande mudança conformacional, expondo o sítio ligante para o lado extracelular. Este modelo, com pequenas variações, é aceito para a maioria das proteínas transportadoras pertencentes à superfamília $\mathrm{ABC} .^{27,28}$

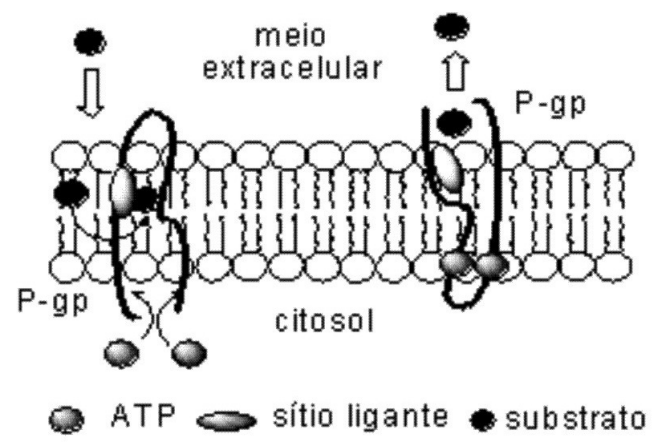

Figura 3. Modelo de Higgins para a atuação da P-gp como flipase

\section{ESTRUTURA DA P-GP E BASE MOLECULAR DA INTERAÇÃO COM OS SUBSTRATOS}

A glicoproteína-P tem uma incomum poliespecificidade, reconhecendo milhares de compostos, variando de 300 a 4000 daltons.

A determinação das estruturas de transportadores da superfamília ABC presentes em bactérias, ${ }^{27,29}$ e a caracterização da P-gp por microscopia eletrônica com baixa resolução ${ }^{30}$ foram avanços importantes para a elucidação completa da estrutura da P-gp. Mais recentemente, Aller et. $a l .{ }^{31}$ descreveram estruturas cristalográficas de uma P-gp de camundongo ligada a substratos, apresentando $87 \%$ de homologia com a P-gp humana. A estrutura da P-gp apresenta-se como um arranjo conformacional de duas metades, cada uma com seis segmentos transmembrana, totalizando $12 \mathrm{TM}$, que entram perpendicularmente na membrana plasmática (136 ̊, perpendicularmente e $70 \AA$, no plano da bicamada), e com um sítio de ligação para nucleotídeo (NBD) em cada metade, na porção citosólica da P-gp, separados por 30 Å. Dois "portais" permitem o acesso direto de moléculas hidrofóbicas a partir da membrana celular. Estes portais são formados pelo segmentos TM4-TM6 e TM10-12. Uma cavidade interna, dentro da bicamada lipídica, com volume bastante substancial ( $6000 \AA)$ pode acomodar, no mínimo, dois substratos simultaneamente. ${ }^{32}$ A região do sítio ligante é formada em sua maioria por resíduos aromáticos e hidrofóbicos. Dos 73 resíduos indicados por acessibilidade de solvente, 15 são polares, sendo que apena 2 (His60 e Glu871), localizados na porção $\mathrm{N}$-terminal, são potencialmente carregados.

A P-gp diferencia estereoisômeros de peptídeos cíclicos, indicando diferentes sítios de ligação. A Figura 4 é um diagrama de Venn, representando as regiões ligantes para os dois estereoisômeros do peptídeo cíclico QZ59 e verapamil. Conforme pode ser observado, os sítios ligantes dos três substratos apresentam em comum dois resíduos: valina (V978) e fenilalanina (F724). Embora alguns resíduos da P-gp possam interagir com ambos os isômeros QZ59, existem regiões de estereoespecificidade: os resíduos aromáticos de tirosina (Y303) e fenilalanina (F728) e de serina (S975) interagem especificamente com o enantiômero $R, R, R$, enquanto aminoácidos não aromáticos predominam na interação específica com o enantiômero $S, S, S$ : leucina (330, 758), glicina (G985), glutamina (Q986) e serina (989). As regiões mais hidrofóbicas encontram-se na porção mais alta da cavidade interna da P-gp (mais próxima à camada mais externa da membrana celular), enquanto a parte mais inferior apresenta resíduos polares (histidina e glutâmico), permitindo a interação com substratos carregados.

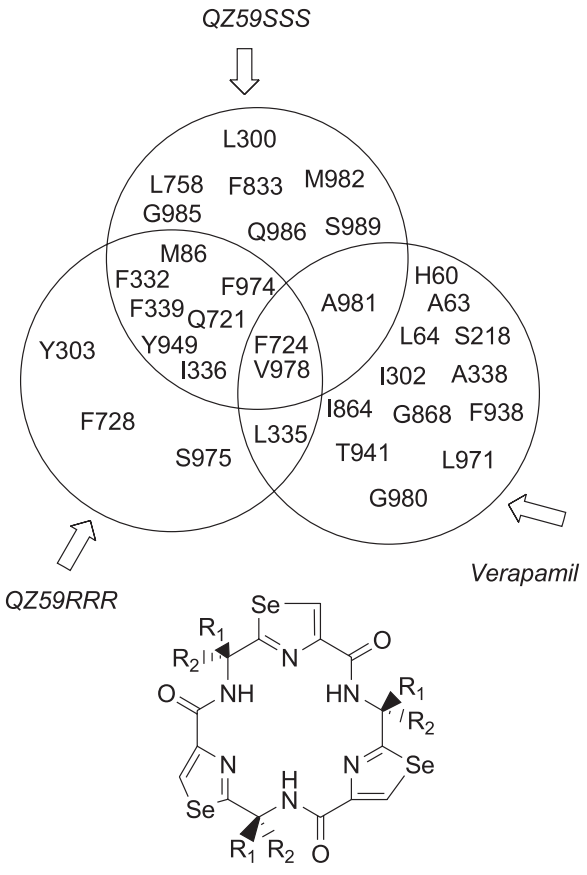

QZ59SSS: $\mathrm{R}_{1}=$ isopropila, $\mathrm{R}_{2}=\mathrm{H}$ QZ59RRR: $R_{1}=H, R_{2}=$ isopropila

Figura 4. Diagrama de Venn representando a relação entre os sítios ligantes da P-gp para QZ59 e verapamil

\section{ESTUDOS DAS RELAÇÕES-ESTRUTURA ATIVIDADE REVERSORA MDR}

Zamora, Pierse e Beck $^{33}$ realizaram um dos primeiros estudos visando estabelecer uma relação entre atividade moduladora da MDR aos alcaloides da Vinca de uma série de compostos com estruturas químicas variadas e algumas de suas propriedades. Os seus coeficientes de partição octanol-água, expressos como logaritmos (LogP e $\log \mathrm{D}$ em $\mathrm{pH} 7,4$, para os compostos ionizáveis) foram medidos e as refratividades molares $\left(\mathrm{R}_{\mathrm{M}}\right)$ calculadas. Os resultados mostraram que os valores de RM eram muito variáveis e que não havia relação direta com a atividade moduladora da MDR. Uma correlação moderada $\left(\mathrm{R}^{2}\right.$ $=0,62$ ) foi obtida entre os valores de $\operatorname{LogD}$ de 17 reversores. Embora neste estudo alguns moduladores altamente eficazes como, por exemplo, a reserpina, tenham sido excluídos, os autores concluíram que a solubilidade lipídica no $\mathrm{pH}$ 7,4 e a presença de formas iônicas protonadas eram características importantes para a atividade anti-MDR.

Em 1997, a primeira análise de QSAR-3D para fenotiazinas e compostos estruturalmente relacionados, conhecidos por inibir a atividade da P-gp, foi descrita. ${ }^{34}$ Após este estudo, vários outros surgiram na literatura e foram extensiva e cuidadosamente discutidos por Wiese e Pajeva. ${ }^{35}$

Apesar da grande variedade estrutural de substratos envolvidos na MDR, é consenso que muitos deles compartilham algumas propriedades comuns. A maioria deles é formada por compostos lipofílicos, porém apresentando grupos que garantam alguma hidrofilicidade. A presença de grupos protonáveis em $\mathrm{pH}$ fisiológico em extensão variável (bases fracas) também é uma característica estrutural frequente nestes compostos. A seguir, apresentamos um resumo dos requisitos estruturais e principais propriedades físico-químicas relacionados à capacidade moduladora da MDR.

\section{Hidrofobicidade}

Esta propriedade parece ser comum entre muitos reversores da MDR. Em vários estudos, foi possível determinar uma boa correlação 
entre coeficiente de partição e atividade anti-MDR, embora a importância da presença de grupos funcionais que não sejam polares não tenha uma relação direta com a partição, mas sim com a interação com regiões hidrofóbicas na P-gp. Assim, a presença de campos hidrofóbicos em estudos de modelagem é muito mais representativa para a atividade anti-MDR do que medidas de coeficiente de partição $(\operatorname{LogP}$ ou $\log D){ }^{3.5}$

\section{Peso molecular e requerimento estérico}

A complementaridade na forma e no tamanho entre um ligante e seu receptor sempre foi considerada como um fator importante para que haja uma interação efetiva ligante-receptor. O mesmo é verdade para as interações entre $\mathrm{P}$-gp e seus potenciais substratos. $\mathrm{O}$ efeito do tamanho da molécula sobre a atividade anti-MDR foi inicialmente estudado em 1970 por Biedler e Riehm ${ }^{36}$ e, 20 anos depois, confirmado por Selassie et al.. ${ }^{37}$

Diferentes parâmetros são utilizados para quantificar as propriedades estéricas de drogas e fármacos, mas a determinação mais elementar é pelo seu peso molecular. Moléculas muito pequenas não são substratos nem inibidores para a P-gp, e o intervalo de peso molecular considerado apropriado é 250-2000. Um estudo baseado na mutação do segmento TM1, no qual o resíduo His61 foi substituído por aminoácidos de cadeias laterais de tamanhos variáveis ${ }^{38}$ sugere que cadeias menores criariam um bolso que permitiria a interação com substratos maiores como, por exemplo, a vimblastina, enquanto os de cadeia maior seriam adequados para a acomodação de substratos menores. O ponto sensível deste estudo é que a modificação na natureza dos aminoácidos presentes neste segmento TM1 poderia levar a mudanças conformacionais e, estas sim, seriam responsáveis pela acomodação de um substrato em detrimento de outro.

A questão do papel do tamanho do potencial substrato para a P-gp sobre a atividade anti-MDR continua em discussão. Os estudos mais recentes na literatura correlacionam o tamanho da molécula com a capacidade de sofrer endocitose $\mathrm{e}^{39}$ e suas propriedades mecânicas da membrana plasmática, ${ }^{40}$ o que não está diretamente relacionado à possibilidade de se ligar ou não a um dos sítios ligantes da $\mathrm{P}$-gp.

\section{Aceptores para ligações de hidrogênio}

A relevância da presença destes aceptores foi comprovada por estudos QSAR-3D, empregando um modelo de análise comparativa de campo molecular (CoMFA). ${ }^{41} \mathrm{Um}$ estudo realizado por Seelig ${ }^{42}$ determinou a importância da presença de múltiplos aceptores, geralmente oxigênio carbonílico e grupos alcóxi, por exemplo metoxilas, separados por uma distância entre 2,5-4,6 ̊, sendo uma característica comum a muitos moduladores da resistência. A importância da presença de grupos HBA (hydrogen bond accepting) é bem discutida na literatura. ${ }^{43}$

\section{Presença de nitrogênio básico}

Dentre os grupos farmacofóricos propostos anteriormente por Zamora et al., ${ }^{33}$ está um átomo de nitrogênio básico. Embora vários moduladores de fato compartilhem esta característica, alguns outros igualmente potentes não a apresentam. ${ }^{44}$ Assim, Chiba et al.${ }^{45}$ investigaram a importância do átomo de nitrogênio na estrutura de uma série de compostos, utilizando como protótipo a molécula da propafenona. Todos os compostos estudados apresentaram uma excelente correlação entre a inibição do efluxo de rodamina 123 e da daunorrubicina $(\mathrm{r}=0,995)$. Como espécies representativas, eles incluíram compostos nitrogenados com grau variável de $\mathrm{pKa}$, dos quais selecionamos alguns exemplos (Figura 5) para ilustrar as conclusões de Chiba e colaboradores: as aminas terciárias GP05, GP29 e GP62, anilinas
(GP240), amidas (GP360) e outros que apresentam mais de um tipo de nitrogênio na mesma estrutura (GP388).

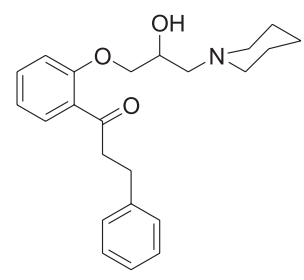

$\mathrm{EC}_{50}($ rodamina 123$)=1,45 \mu \mathrm{mol} / \mathrm{L}$ $\mathrm{EC}_{50}$ (daunorrubicina) $=0,65 \mu \mathrm{mol} / \mathrm{L}$

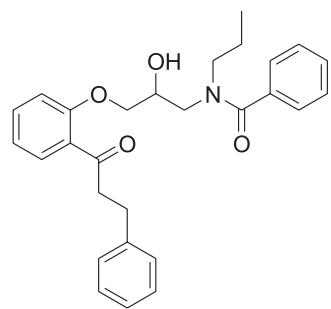

GP $360, \mathrm{pKa}=-1,46$

$\mathrm{EC}_{50}($ rodamina 123$)=2,57 \mu \mathrm{mol} / \mathrm{L}$ $\mathrm{EC}_{50}$ (daunorrubicina) $=1,65 \mu \mathrm{mol} / \mathrm{L}$

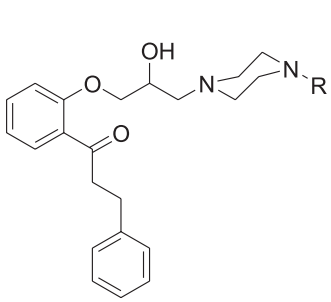

GP 29, $(\mathrm{R}=$ benzila); $\mathrm{pKa}=7,45$

$\mathrm{EC}_{50}$ (rodamina 123$)=0,62 \mu \mathrm{mol} / \mathrm{L}$

$\mathrm{EC}_{50}$ (daunorrubicina) $=0,39 \mu \mathrm{mol} / \mathrm{L}$
GP 05, pKa $=8,44$

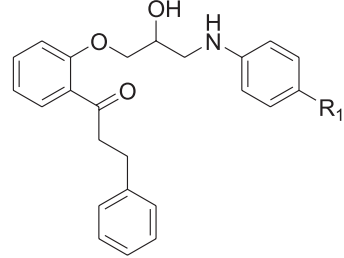

GP $240\left(R_{1}=H\right), p K a=3,25$

$E_{50}($ rodamina 123$)=17,61 \mu \mathrm{mol} / \mathrm{L}$ $\mathrm{EC}_{50}$ (daunorrubicina) $=6,47 \mu \mathrm{mol} / \mathrm{L}$

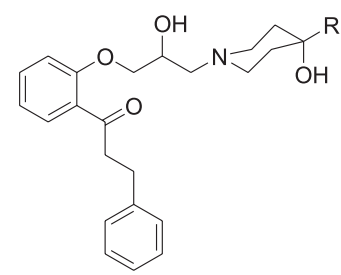

GP 62, $(R=$ fenila); $p K a=7,56$ $\mathrm{EC}_{50}$ (rodamina 123) $=0,11 \mu \mathrm{mol} / \mathrm{L}$ $\mathrm{EC}_{50}$ (daunorrubicina) $=0,06 \mu \mathrm{mol} / \mathrm{L}$
Figura 5. Alguns compostos nitrogenados usados em estudo para determinar a importância do nitrogênio

A partir da análise da Figura 5, podemos observar que não há uma relação direta entre a diminuição da basicidade do nitrogênio e o poder inibitório do efluxo da rodamina 123. Embora a diminuição do pKa de GP05 para GP240 tenha sido acompanhada da diminuição da inibição do efluxo (aumento do $\mathrm{EC}_{50}$ ), o mesmo não é observado, comparando-se GP05 e GP360. Neste caso, a menor basicidade foi superada pela introdução de um grupamento funcional que pode participar de ligações de H. GP29 e GP388 se diferenciam pela presença da carbonila neste último, mudança estrutural que foi acompanhada por um significativo aumento no poder inibitório do efluxo de rodamina. A importância da presença de grupos participantes de ligações de hidrogênio fica ainda mais patente quando da observação do efeito antiMDR de GP62. Embora os autores afirmem neste trabalho que a presença de um nitrogênio ionizável não é requisito para a atividade, é necessário ter certa cautela nestas conclusões, pois o composto mais ativo, GP62, além de apresentar o grupo hidroxila, tem também um átomo de nitrogênio ionizável. A amida GP360 não é a mais ativa, mesmo tendo o grupo carbonila.

\section{Experimentos para investigação da atividade moduladora da MDR}

Ensaios de inibição da proliferação celular

A citotoxicidade do candidato a modulador, expressa através da concentração inibitória mínima necessária para inibir em 50\% o 
crescimento celular $\left(\mathrm{CI}_{50}\right.$ ou $\left.\mathrm{IC}_{50}\right)$, deve ser medida. Inicialmente se faz a avaliação da atividade antiproliferativa sobre a linhagem estudada, para que um eventual efeito inibidor da proliferação promovido pela própria substância-teste não dê um resultado falso-positivo para a reversão da resistência ao quimioterápico, além de permitir a determinação da concentração sub-tóxica para a linhagem. O verapamil é o controle positivo utilizado e, em alguns casos, a reserpina.

Dois protocolos são conhecidos: um, em que as células são incubadas previamente na presença do candidato a inibidor, em diferentes concentrações, e, após um período que varia de 1 a 3 h, o quimioterápico ao qual a linhagem é resistente, geralmente doxorrubicina ou vimblastina, é adicionado e a contagem de células ou a leitura do conteúdo proteico é feita $48 \mathrm{~h}$ depois. Em outro protocolo, não há uma pré-exposição das células ao potencial "sensibilizador", e o que se faz é adicionar simultaneamente o quimioterápico e a substância teste. Em ensaios preliminares, a proporção substância teste/quimioterápico é de 1:1 e, com base no resultado obtido, a proporção poderá ser aumentada, caso não se observe nenhum efeito, ou diminuída, se a linhagem tiver sido sensibilizada, até que se determine a proporção mínima para observação do efeito. ${ }^{46}$

\section{Acúmulo de quimioterápico ou captação da droga}

Uma vez que tenha sido observada a sensibilização da linhagem celular ao quimioterápico, é preciso demonstrar que o efeito é devido ao aumento do acúmulo do quimioterápico no interior das células. Em geral, as células são pré-incubadas com a substância teste, por 15 min, a $37{ }^{\circ} \mathrm{C}$. Em seguida, daunorrubicina ou outro substrato fluorescente para P-gp é adicionado e após cerca de $180 \mathrm{~h}$ a fluorescência intracelular é medida. ${ }^{47} \mathrm{O}$ método não deve ser utilizado com qumioterápicos de baixa fluorescência. Em geral, doxorrubicina (adriamicina) é utilizada e a fluorescência é medida em citômetro de fluxo. ${ }^{48}$

Ao final do experimento compara-se a reversão (índice de modulação) com o aumento da captação do quimioterápico para determinar a dependência do processo.

\section{Inibição do efluxo pela P-gp}

Esta é uma medida de afinidade da substância teste pela P-gp. A síntese e uso de análogos fotoativos de agentes citotóxicos têm permitido a identificação da P-gp como o alvo de alguns reversores da MDR. Análogos tritiados ou derivados iodados $\left({ }^{125} \mathrm{I}\right)$ da vimblastina, de antraciclinas e do paclitaxel vem sendo utilizados, além do corante rodamina $123 .{ }^{49}$ A linhagem Caco-2 é empregada nestes experimentos de transporte, e os protocolos estão descritos na literatura. ${ }^{50}$

\section{Medidas para expressar a atividade reversora}

Na modulação farmacológica da resistência são usados vários critérios para identificar e avaliar a atividade antirresistência, cada um fornecendo informações diferentes na atividade de modulação e tendo suas próprias vantagens e desvantagens. Em geral são:

\section{Razão $M D R$}

Também conhecida como razão de modulação, razão de sensibilização ou ainda grau de potencialização, é definida pela razão entre o valor de $\mathrm{IC}_{50}$ do agente citotóxico na ausência do modulador $\left(\mathrm{IC}_{50} \mathrm{q}\right)$ e o $\mathrm{IC}_{50}$ na presença do mesmo ( $\mathrm{IC}_{50 \mathrm{qm}}$, Equação 1).

$$
\text { Razão MDR }=\frac{I C_{q}}{I C_{q m}}
$$

Esse critério, usado na maioria dos estudos de modulação da resistência, é uma avaliação da citotoxicidade geral do agente antitumoral sobre uma determinada linhagem celular, quando um modulador da resistência é empregado. A razão pode variar substancialmente para o mesmo modulador dependendo da linhagem celular tumoral e do quimioterápico em questão. Por exemplo, a razão MDR é por volta de doze vezes maior na linhagem MFC-7 do que em P388, quando doxorrubicina e transflupentixol são usados como quimioterápico e modulador, respectivamente. Ao mesmo tempo, razões quase equivalentes são observadas para as linhagens KB e MCF-7 quando vimblastina é o quimioterápico utilizado para a primeira linhagem e doxorrubicina para a segunda e o mesmo trans-flupentixol é usado, sugerindo alguma similaridade na forma como este modulador quimiossensibiliza essas linhagens celulares. ${ }^{51}$

\section{Índice do modulador (MI ou IM)}

Também chamado de índice de modulação, o conceito foi introduzido para solucionar o problema da influência da concentração do modulador, que não é considerada quando a modulação é expressa em razão mdr. Ele é definido como o quociente da razão MDR e a concentração molar do modulador (Equação 2). Entretanto, o uso do conceito MI deve ser cuidadoso, pois ele sugere uma relação linear entre razão MDR e concentração molar do modulador. Pearce e colaboradores ${ }^{52}$ observaram que os valores de MI calculados para três diferentes concentrações do mesmo modulador apresentavam variações de até 10 vezes o valor.

$$
\mathrm{MI}=\frac{\text { razão MDR }}{\text { [modulador] }}
$$

\section{Inibidores de terceira geração}

Muitas drogas e fármacos vêm sendo investigados nos últimos anos para modular o transporte de quimioterápicos pela $\mathrm{P}$-gp e, dentre eles, bloqueadores de canais de cálcio, sendo o verapamil o principal representante, como já comentado. Estes compostos não foram desenvolvidos especificamente para bloquear a P-gp, e muitos deles são substratos para outros transportadores e sistemas enzimáticos, resultando em interações farmacocinéticas não previsíveis. Além disso, muitos destes inbidores da P-gp só o fazem em doses bastante altas, apresentando muitas vezes um nível de toxicidade bastante elevado. ${ }^{53}$

A segunda geração de moduladores inclui o biricodar, valspodar, dexverapamil, dexniguldipina, e derivados da indolizilsulfona e triazinoaminopiperidina ${ }^{53-55}$ Estes, compostos, embora mais potentes e menos tóxicos que os de primeira geração, apresentam algumas limitações significativas. ${ }^{56}$ Por exemplo, biridacor e valspodar inibem o metabolismo do quimioterápico paclitaxel, via citocromo P450A4.

Face aos problemas relacionados à farmacocinética, especificidade e toxicidade, a literatura vem sendo contemplada com muitas publicações que reúnem resultados de pesquisas voltadas para o desevolvimento de novos inibidores, variando desde a investigação de requisitos estruturais mínimos, proposição de modelos farmacofóricos, mapas de auto-organização para identificação de inibidores, ${ }^{57}$ até a seleção de modelos a partir de bibliotecas. ${ }^{58}$

Muitos destes inibidores investigacionais empregam o verapamil como protótipo, geralmente mantendo-se o grupo dimetoxifeniletilamina. As succinimidas I e os aminoésteres II e III (Figura 6) são alguns exemplos.

As succinimidas I foram mais potentes que o verapamil em inibir o efluxo pela P-gp. A razão de atividade de florescência da succinimida Ia foi o dobro da exibida pelo verapamil, em uma concentração 10 vezes menor. ${ }^{59}$ Estes resultados parecem estar relacionados com as interações $\pi$ que os dois anéis aromáticos do sistema policíclico poderiam estabelecer com a proteína, além da presença de grupos HBA (hydrogen bond accepting). Os aminoésteres II e III também 


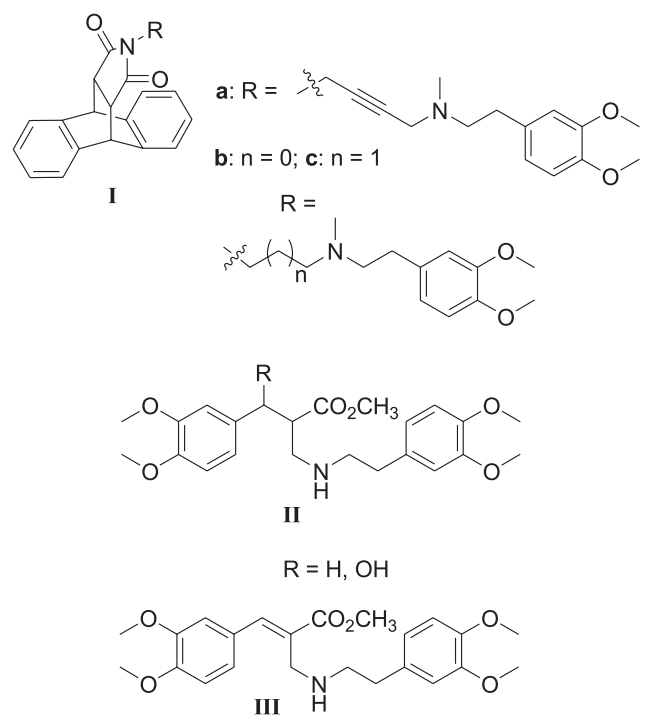

Figura 6. Moduladores da MDR baseados na estrutura do verapamil

compartilham estas características, sendo que III foi o derivado mais potente, cerca de 6 vezes o verapamil. ${ }^{60}$

A terceira geração de moduladores é constituída por inibidores como, por exemplo, o elacridar e tariquidar (Figura 7), que não apresentam interações farmacocinéticas com quimioterápicos e mostraram alta potência e especificidade para a P-gp. Elacridar ${ }^{61,62}$ e tariquidar ${ }^{63,64}$ encontram-se em fase I e III de estudo, respectivamente.

Embora estas substâncias compartilhem a porção dimetoxitetraidroisoquinolina ligada a um grupo anillida por uma ponte etilênica, os estudos das relações estrutura-atividade não têm esclarecido com segurança a contribuição de cada um destes grupos para reverter a MDR. Assim, muitos outros derivados vêm sendo investigados. ${ }^{65}$

Recentemente, uma estratégia diferente para a modulação da MDR surgiu na literatura. Após a descoberta de que alguns excipientes e aditivos comumente utilizados em formulações farmacêuticas poderiam inibir a função da P-gp intestinal, Talegaonkar et.al. ${ }^{66}$ vislumbraram a possibilidade de desenvolver novos sistemas de liberação de fármacos (DDS) para modular a resistência a múltiplas drogas mediada pela P-gp. Dentre estes sistemas se encontram microsferas, lipossomas e nanopartículas.

Sabendo que um determinado excipiente ou aditivo não sofre extrusão pela P-gp, encapsular o quimioterápico despontou como uma possibilidade do fármaco passar pela membrana plasmática sem ser reconhecido pela P-gp, chegando ao citoplasma da célula, onde é liberado. Quimioterápicos como o paclitaxel ${ }^{67}$ e doxorrubicina ${ }^{68}$ têm sido encapsulados com base nesta estratégia, que também vem sendo empregada para a liberação de moduladores, como o tariquidar, um modulador de terceira geração. ${ }^{69}$

\section{CONCLUSÕES}

A resistência a múltiplas drogas (MDR) é um grande problema na terapia do câncer. Esta é um tipo de resistência adquirida, mas que pode ser revertida por fármacos ou drogas conhecidos como moduladores, reversores ou, ainda, sensibilizadores. Embora existam vários mecanismos pelos quais a reversão pode ocorrer, o principal e o mais estudado é via proteínas transportadoras de membrana, cuja atividade promove o efluxo do quimioterápico, evitando que este atinja uma concentração intracelular terapêutica. Existem várias proteínas envolvidas neste processo de efluxo do fármaco, mas a principal é glicoproteína-P (P-gp), que se tornou um alvo importante para o desenvolvimento de agentes moduladores da MDR. Os moduladores

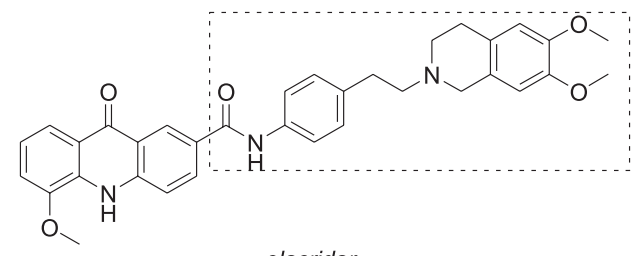

elacridar

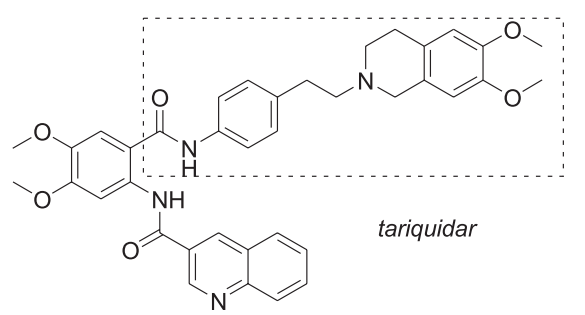

Figura 7. Elacridar e Tariquidar, moduladores de $3^{a}$ geração

de $1^{a}$ geração são fármacos conhecidos pelo seu uso terapêutico, tais como alguns bloqueadores de canais de cálcio, sendo o verapamil o mais importante. Uma vez que as doses necessárias para a reversão são muito altas, o aparecimento de efeitos indesejados e interações farmacocinéticas levaram à busca de outros moduladores, utilizando como protótipo a estrutura do verapamil. Embora os estudos SAR não sejam conclusivos, a busca pelo estabelecimento de um ou mais modelos farmacofóricos pode minimizar as dificuldades na descoberta de novos moduladores da MDR. Uma boa parte desta pesquisa foi facilitada pela determinação estrutural da P-gp, assim como pelo conhecimento de seus sítios ligantes. A importância de algumas características estruturais e propriedades têm sido apontadas e estão presentes na grande maioria dos moduladores. Peso molecular entre 250 e 500, lipofilicidade e grupos aceptores de hidrogênio mantendo uma distância de 2,5-4,6 ̊̊ são as principais características.

Atualmente, dois moduladores da MDR, baseados na inibição da P-gp estão em fase avançada de estudo: o elacridar (fase I) e o tariquidar (fase III). Estratégias baseadas em formulações farmacêuticas também são empregadas e são objeto de patenteamento e publicações científicas, em número crescente.

A literatura referente às implicações da glicoproteína-P no fenômeno de resistência a múltiplas drogas é rica, crescente, cobrindo diferentes aspectos da pesquisa no campo da MDR envolvendo a glicoproteína-P, tais como estudos cinéticos, ${ }^{70}$ descoberta de novos agentes moduladores naturais ${ }^{71}$ e sintéticos ${ }^{72}$ e aplicações potenciais da inibição da atividade da P-gp. ${ }^{73}$

\section{REFERÊNCIAS}

1. Longley, D. B.; Johnston, P. G.; J. Pathol. 2005, 205, 275.

2. Hill, B.; Int. J. Oncol. 1996, 9, 197.

3. Mitscher, L. A.; Pillai, S. P.; Gentry, E. J.; Shankel, D. M.; Med. Res. Rev. 1999, 19, 477.

4. Dewick, P. M.; Medicinal Natural Products, a Biosynthetic Approach, $3^{\text {rd }}$ ed., Wiley: United Kingdom, 2009.

5. Dietel, M.; Pathol. Res. Pract. 1991, 187, 892.

6. Beck, W. T.; Cancer Treat. Rev. 1990, 17, 11.

7. Morrow, C. S.; Cowan, K. H.; Cancer Cell 1990, 2, 15.

8. Hammond, J. R.; Johnstone, R. M.; Gros, P.; Cancer Res. 1989, 49, 3867.

9. Hannun, Y. A.; Blood 1977, 89, 1845.

10. Szakacs, G.; Paterson, J. K.; Ludwig., J. A.; Booth-Genthe, C.; Gottesman, M. M.; Nat. Rev. Drug Discovery 2006, 5, 219.

11. Kessel, D.; Bottenrill, V.; Wodinsky, I.; Cancer Res. 1968, 28, 938.

12. Jones, P. M.; George, A. M.; J. Membr. Biol. 1998, 166, 133. 
13. Robert, J.; Drugs Fut. 1997, 22, 149.

14. Juliano, R. L.; Ling, V.; Biochim. Biophys. Acta 1976, 455, 152.

15. Ruetz, S.; Gros, P.; Cell 1994, 77, 1071.

16. Borst, P.; Evers, R.; Kool, M.; Wijnholds, J.; J. Nat. Cancer Inst. 2000, 92, 1295.

17. Hipfner, D. R.; Deeley, R. G.; Cole, S. P. C.; Biochim. Biophys. Acta 1999, 1461, 359.

18. Litman, T.; Druley, T. E.; Stein, W. D.; Bates, S. E.; Cell. Mol. Life Sci. 2001, 58, 931 .

19. Deeley, R. G.; Cole, S. P. C.; Cancer Biol. 1997, 8, 193.

20. Gatti, L.; Beretta, G. L.; Cossa, G.; Zunino, F.; Perego, P.; Mini-Rev. Med. Chem. 2009, 9, 1102.

21. Gottesmann, M. M.; Pastan, I.; Annu. Rev. Biochem. 1993, 62, 385.

22. King, M.; Su, W.; Chang, A.; Zuckerman, A.; Pasternak, G. W.; Nature Neurosci. 2001, 4, 268.

23. Liu, X. D.; Liu, G. Q.; Acta Pharmacol. Sin. 2001, 22, 111.

24. Raviv, Y.; Pollard, H. B.; Bruggemann, E. P.; Pastan, I.; Gottesmann, M. M.; J. Biol. Chem. 1990, 265, 3975.

25. Stein, W. D.; Phys. Rev. 1977, 77, 545.

26. Higgins, C. F.; Cell 1994, 79, 393.

27. Dawson, R. J. P.; Locher, K. P.; Nature 2006, 443, 180.

28. Stenham, D. R.; Campbell, J. D.; Sansom, M. S. P.; Higgins, C. F.; Kerr, I. D.; Linton, K. J.; FASEB J. 2003, 17, 2287.

29. Locher, K. P.; Lee, A. T.; Rees, D. C.; Science 2002, 296, 1091; Hollenstein, K.; Frei, K. P.; Locher, K. P.; Nature 2007, 446, 213; Pinkett, H. W.; Lee, A. T.; Lum, P.; Locher K. P.; Rees, D. C.; Science 2007, 315, 373; Oldham, M. L.; K.hare, D.; Quiocho, F. A.; Davidson, A. L.; Chen, J.; Nature 2007, 450, 515.

30. Rosenberg, M. F.; Kamis, A. B.; Callaghan, R.; Higgins, C. F.; Ford, J.; J. Biol. Chem. 2003, 278, 8294; Lee, J.-Y.; Urbatsch, I. L.; Senior, A. E.; Wilkens, S.; J. Biol. Chem. 2008, 283, 5769.

31. Aller, S. G.; Yu, J.; Ward, A.; Weng, Y.; Chittaboina, S.; Zhuo, R.; Harrell, P. M.; Trinh, Y. T.; Zhang, Q.; Urbatsch, I. L.; Chang, G.; Science 2009, 323, 1718.

32. Loo, T. W.; Bartlett, M. C.; Clarke, D. M.; J. Biol. Chem. 2003, 278, 39706.

33. Zamora, J. M.; Pearce, H. L.; Beck, W. T.; Biochem. Pharmacol. 1988, $33,454$.

34. Pajeva, I. K.; Wiese, M.; QSAR 1997, 16, 1.

35. Wiese, M.; Pajeva, I. K.; Curr. Med. Chem. 2001, 8, 685.

36. Biedler, J. R.; Riehm, H.; Cancer Res. 1970, 30, 1174.

37. Selassie, C. D.; Hansch, C.; Khwaja, T. A.; J. Med. Chem. 1990, 33, 1914.

38. Ueda, K.; Taguchi, Y.; Morishima, M. S.; Cancer Biol. 1997, 8, 151.

39. Rauch, C.; Farge, E.; Biophys. J. 2000, 78, 3036; Rauch, C.; Pluen, A.; Eur. Biophys. J. 2007, 36, 121

40. Rauch, C.; Eur. Biophys. J. 2009, 38, 537.

41. Ekins, S.; Kim, R. B.; Leake, B. F.; Dantzig, A. H.; Schuetz, E. G.; Lan, L.-B.; Yasuda, K. Y.; Shepard, R. L.; Winter, M. A.; Schuetz, J. D.; Wikel, J. H.; Wrighton, S. A.; Mol. Pharmacol. 2002, 61, 964.

42. Seelig, A.; Int. J. Clin. Pharmacol. Ther. 1998, 36, 54.

43. Robert, J.; Jarry, C.; J. Med. Chem. 2003, 46, 4805.

44. Ueda, K.; Okamura, N.; Hirai, M.; Tanigawara, Y.; Saeki, T.; Kioka, N.; Komano, T.; Hori, R.; J. Biol. Chem. 1992, 267, 24248.

45. Ecker, G.; Huber, M.; Schmid, D.; Chiba, P.; Mol. Pharmacol. 1999, 56, 791.

46. Um exemplo de protocolo pode ser encontrado em Gopinath, V. S.; Thimmaiah, P.; Thimmaiah, K. N.; Bioorg. Med. Chem. 2008, 16, 474.
47. Jekerle, V.; Klinkhammer, W.; Scollard, D. A.; Breitbach, K.; Reilly, R. M.; Piquette-Miller, M.; Wiese, M.; Int. J. Cancer 2006, 119, 414; Ramalhete, C.; Molnar, J.; Silva, M.; Rosário, V. E.; Ferreira, M-J. U.; Bioorg. Med. Chem. 2009, 17, 6942.

48. Dhainaut, A.; Regnier, G.; Atassi, C.; Pierre, A.; Leonce, S.; KrausBeRthier, L.; Prost, J.-F.; J. Med. Chem. 1992, 35, 2481.

49. Safa, A. R.; Drug Res. Updates 1999, 2, 371.

50. Taub, M .E.; Podila, L.; Almeida, I.; Drug Metab. Dispos. 2005, 33, 1679.

51. Ford, J. M.; Bruggeman, E. P.; Pastan, I.; Gottesmann, M.; Hait, W. N.; Cancer Res. 1990, 50, 1748.

52. Pearce, H. L.; Winter, M. A.; Beck, W. T.; Adv. Enzyme Regul. 1990, 30, 357.

53. Thomas, H.; Coley, H. M.; Cancer Control 2003, 10, 159.

54. Dey, S.; Curr. Opin. Inv. Drugs 2000, 3, 818.

55. Rago, R. P.; Einstein, A.; Lush, R.; Beer, T. M.; Ko, Y. J.; Henner, W. D.; Bubley, G.; Merica, E. A.; Garg, V.; Ette, E.; Harding, M. V.; Dalton, V. S.; Cancer Chemother. Pharmacol. 2003, 51, 297.

56. Minderman, H.; O’Loughlin, K. L.; Pendyala, L.; Baer, M. R.; Clin. Cancer Res. 2004, 10, 1826.

57. Kaiser, D.; Terfloth, L.; Kopp, S.; Schulz, J.; de Laet, R.; Chiba, P.; Ecker, G. F.; Gasteiger, J.; J. Med. Chem. 2007, 50, 1698.

58. Ferrer-Montiel, A.; Messeguer, A.; Ferragut, J. A.; Pérez-Payá, E.; Masip, I.; Cortés, N.; Abdad-Merin, M. J.; J. Pharmacol. Exp. Ther. 2005, 313, 112.

59. Bisi, A.; Gobbi, S.; Rampa, A.; Belluti, F.; Piazzi, L.; Valenti, P.; Gyemant, N.; Molnár, J.; J. Med. Chem. 2006, 49, 3049.

60. Huber, P.C.; Pettenazzi, M. C. R.; Kohn, L. K.; Tormena, C. F.; Almeida, W. P.; 10 $10^{\text {th }}$ Tetrahedron Symposium, Paris, France, 2009.

61. Traunecker, H. C.; Stevens, M. C.; Kerr, M. C.; Ferry, D. R.; Br. J. Cancer 1999, 81, 942.

62. Sparreboom, A.; Plantings, A. S.; Jewell, R. C.; van der Gaast, A.; de Bruijn, P.; Loos, W. J.; Nooter, K.; Chandler, L. H.; Paul, E. M.; Wissel, P. S.; Verweij, J.; Anti-Cancer Drugs 1999, 10, 719.

63. Pusztai, L.; Wagner, P.; Ibrahim, N.; Rivera, E.; Theriault, R.; Booser, D.; Symmans, F. W.; Wong, F.; Blumenschein, G.; Fleming, D. R.; Rouzier, R.; Boniface, G.; Hortobagyi, G. N.; Cancer 2005, 104, 682; Walker, J.; Martin, C.; Callaghan, R.; Eur. J. Cancer 2004, 40, 594 e referências citadas.

64. McDevitt, C. A.; Callaghan, R.; Pharmacol. Ther. 2007, 113, 429.

65. Colafubo, N. A.; Berardi, F.; Cantore, M.; Perrone, M. G.; Contino, M.; Inglese, C.; Niso, M.; Perrone, R.; Azzariti, A.; Simone, G. M.; Porcelli, L.; Paradiso, A.; Bioorg. Med. Chem. 2008, 16, 362.

66. Talegaonkar, S.; Khar, R. K.; Jaggi, M.; Akhtar, N.; Bansal, T.; DDT 2009, 14, 1067 .

67. Chavanpatil, M. D.; Patil, Y.; Panyam, J.; Int. J. Pharm. 2006, 320, 150.

68. Chavanpatil, M. D.; Khdair, A.; Gerard, B.; Bachmeier, C.; Miller, D. W.; Shekhar, M. P. V.; Panyam, J.; Mol. Pharmacol. 2007, 4, 730.

69. Patil, Y.; Sadhukha, T.; Ma, L.; Panyam, J.; J. Controlled Release 2009, $136,21$.

70. Tachibana, T., Kitamura S.; Kato, M.; Mitsui, T.; Shirasaka, Y.; Yamashita, S.; Sugiyama, Y.; Pharm. Res. 2010, 27, 442; Siarheyeva, A.; Liu, R.; Sharom, F. J.; J. Biol. Chem., 2010, 285, 7575.

71. Borska, S.; Sopel, M.; Chmielewska, M.; Zabel, M.; Dziegiel, P.; Molecules 2010, 15, 857; Sun, L.-R.; Cui, S.-X.; Qu, X.-J.; Drugs Fut. 2010, 34, 53; Riveiro, M. E.; De Kimpe, N.; Moglioni, A.; Vazquez, R.; Monczor, F.; Shayo, C.; Davio, C.; Curr. Med. Chem. 2010, 17, 1325.

72. Singh, P.; Kaur, J.; Bhardwaj, A.; Eur. J. Med. Chem. 2010, 45, 1256.

73. Colabufo, N. A.; Berardi, F.; Cantore, M.; Contino, M.; Inglese, C.; Niso, M.; Perrone, R.; J. Med. Chem. 2010, 53, 1883. 